A.-M. Aubert

Nagoya Math. J.

Vol. 170 (2003), 47-72

\title{
CHARACTER SHEAVES AND GENERALIZED SPRINGER CORRESPONDENCE
}

\author{
ANNE-MARIE AUBERT
}

\begin{abstract}
Let $G$ be a connected reductive algebraic group over an algebraic closure of a finite field of characteristic $p$. Under the assumption that $p$ is good for $G$, we prove that for each character sheaf $A$ on $G$ which has nonzero restriction to the unipotent variety of $G$, there exists a unipotent class $C_{A}$ canonically attached to $A$, such that $A$ has non-zero restriction on $C_{A}$, and any unipotent class $C$ in $G$ on which $A$ has non-zero restriction has dimension strictly smaller than that of $C_{A}$.
\end{abstract}

\section{$\S 1$. Introduction}

Let $\overline{\mathbb{F}}_{q}$ be an algebraic closure of a finite field $\mathbb{F}_{q}$ of $q$ elements. Let $G$ be a connected reductive algebraic group over $\overline{\mathbb{F}}_{q}$, defined over $\mathbb{F}_{q}$. Let $T$ be a maximal torus in $G, T^{*}$ be a maximal torus in the Langlands dual $G^{*}$ of $G$ which is dual to $T$, and let $W=W^{G}$ denote the Weyl group of $G$ with respect to $T$, that we identify with the Weyl group of $G^{*}$ with respect to $T^{*}$. Let $s \in T^{*}$. Lusztig has defined a canonical surjective map from the set $\hat{G}$ of characters sheaves on $G$ to the set of $W$-orbits on $T^{*}$. We will denote by $\hat{G}_{s}$ the set of character sheaves in the fibre over the orbit of $s$ of this map. We set

$$
W_{s}^{G}=W_{s}:=\{w \in W \mid w(s)=s\} .
$$

Lusztig has also defined a map from $\hat{G}_{s}$ to the set of two-sided cells of $W_{s}$. For $\mathbf{c}$ a given two-sided cell of $W_{s}$, we will denote by $\hat{G}_{s, \mathbf{c}}$ the set of character sheaves in the fibre over $\mathbf{c}$ of this map. Lusztig has described a canonical construction by which we can associate a well-defined unipotent class $C_{s, \mathbf{c}}$ with each pair $(s, \mathbf{c})$, where $s \in T^{*}$ and $\mathbf{c}$ is a two-sided cell in $W_{s}$. The following theorem is a special case of a result of Lusztig [17, Th. 10.7]. One of our purposes is to replace, in this special case, the assumption

Received July 11, 1997.

Revised February 22, 2000, February 13, 2001, September 11, 2001 and April 1, 2002. 1991 Mathematics Subject Classification: 20G05. 
of $[$ loc. cit. $]$ that the characteristic $p$ of $\mathbb{F}_{q}$ is large enough by the weaker assumption " $p$ is good for $G$ ".

TheOREm 1.1. For any $s \in T^{*}$ and any two-sided cell $\mathbf{c}$ in $W_{s}$, the following hold:

(a) If $A \in \hat{G}_{s, \mathbf{c}}$ and if $C$ is a unipotent class in $G$ such that the restriction of $A$ to $C$ does not vanish, then

$$
\operatorname{dim} C \leq \operatorname{dim} C_{s, \mathbf{c}}, \quad \text { with equality only for } C=C_{s, \mathbf{c}} ;
$$

(b) There exists some $A \in \hat{G}_{s, \mathbf{c}}$ such that the restriction of $A$ to $C_{s, \mathbf{c}}$ does not vanish.

We will first show that part (b) of the Theorem is implied by part (a): for this we will use in a crucial way a result of Geck [5] which generalizes to good primes the result of Lusztig concerning the unipotent support (as an average value) of the irreducible characters of $G^{F}$ and the result of Shoji [22] on Lusztig conjecture. When $G$ is of exceptional type, and, also in some cases for classical groups, we will derive directly the proof of part (a) from Lusztig's one. For the other cases, we will prove it using direct computations in the spirit of [15] in order to obtain a unipotent class $C_{A}$ satisfying analogous statements as (a) and (b), and then use Lusztig's result to check that $C_{A}=C_{s, \mathbf{c}}$. The scheme of the proof is explained at the beginning of Section 4.1 and in (4.1.2).

Acknowledgements. I owe a deep debt of gratitude to the referee for many substantial comments which have strongly influenced this paper.

Part of the paper has been written while I was participating in the Summer School and Workshop on Springer Correspondence and applications in Picquigny in September 1998. I would like to thank the organizers, François Digne, Meinolf Geck, Jean Michel and Alexander Zimmermann, for this invitation and the monastery Notre Dame du Gard for its hospitality.

I gave lectures on this work at the University of Toronto in August 1998, at the University of Poitiers in November 1998, and at Institute Henri Poincaré in February 1999. It is a pleasure to thank Fiona Murnaghan, Pierre Torasso and the Chevalley Seminar for their invitations.

I would like to thank James Arthur, Clifton Cunningham, Meinolf Geck, Philip Kutzko, George Lusztig, Lawrence Morris, Toshiaki Shoji, and JeanLoup Waldspurger for helpful remarks and stimulating discussions in the course of working on this paper. 


\section{§2. Preliminaries}

We keep the notation of the introduction. Let $W_{s}^{0}$ be the Weyl group of the connected component $\mathrm{C}_{G^{*}}^{0}(s)$ of the centralizer $\mathrm{C}_{G^{*}}(s)$ of $s$ in $G^{*}$. It is a normal subgroup of the group $W_{s}$ defined in (1.1). Let $\Phi_{s}$ denote the root system of $W_{s}^{0}$. Let $V$ be the rational vector space spanned by $\Phi_{s}$. Lusztig has associated (see [9], [12, (4.1.2)]) with any irreducible representation $E^{\prime}$ of $W_{s}^{0}$ an integer $b_{E^{\prime}} \geq 0$ by the requirement that $b_{E^{\prime}}$ is the smallest integer $i$ such that $E^{\prime}$ occurs in the $i$-th symmetric power of $V$, and (see [9], [12, (4.1.1)]) with any irreducible representation $E^{\prime}$ of $W_{s}^{0}$, an integer $a_{E^{\prime}} \geq 0$ and an integer $f_{E^{\prime}}>0$ by the requirement that the generic degree of the representation $E^{\prime}$ is of the form

$$
D_{E^{\prime}}(X)=f_{E^{\prime}}^{-1} X^{a_{E^{\prime}}}+\text { higher powers of X. }
$$

Let $E^{\prime}$ be an irreducible representation of $W_{s}$, and let $\tilde{E}^{\prime}$ be an irreducible representation of $W_{s}^{0}$ which occurs in the restriction of $E^{\prime}$ to $W_{s}^{0}$. We will set $a_{E^{\prime}}:=a_{\tilde{E}^{\prime}}$. It does not depend on the choice of the representation $\tilde{E}^{\prime}$, see also $[14,(16.5)]$. For each two-sided cell $\mathbf{c}$, the function $E^{\prime} \mapsto a_{E^{\prime}}$ is constant on the set of irreducible representations $E^{\prime}$ of $W_{s}$ which belong to $\mathbf{c}$, see $[12,(4.14 .1)$ and $(5.25)]$. For any such representation $E^{\prime}$, we will set $a_{s, \mathbf{c}}:=a_{E^{\prime}}$.

Let $\mathcal{N}_{G}$ be the set of pairs $(C, \mathcal{E})$ such that $C$ is a unipotent conjugacy class in $G$, and $\mathcal{E}$ is a $G$-equivariant irreducible local system on $C$, given up to isomorphism. Let $C$ be a unipotent class in $G$, and fix $u \in C$. Then the isomorphism classes of irreducible $G$-equivariant $\overline{\mathbb{Q}}_{\ell}$-local systems on $C$ are in bijection with the isomorphism classes of irreducible representations of the group $A_{G}(u):=\mathrm{C}_{G}(u) / \mathrm{C}_{G}^{0}(u)$ of components of the centralizer $\mathrm{C}_{G}(u)$ in $G$ of $u$. Thus, we can identify a pair $(C, \mathcal{E}) \in \mathcal{N}_{G}$ with the $G$-conjugacy class $[u, \rho]_{G}$ of a pair $(u, \rho)$ where $u$ is a unipotent element in $G$ and $\rho$ is an irreducible representation of the group $A_{G}(u)$. The Springer correspondence is an injective map $\nu_{T}^{G}$ from the set of isomorphism classes of irreducible representations of $W$ into $\mathcal{N}_{G}$. Not all pairs $(C, \mathcal{E})$ in $\mathcal{N}_{G}$ occur in that correspondence.

Let $\mathbf{c}$ be a two-sided cell in $W_{s}$, and $E^{\prime}(\mathbf{c})$ the unique special representation of $W_{s}$ which belongs to $\mathbf{c}$, see $[17,10.4]$. Let $\tilde{E}^{\prime}(\mathbf{c})$ be an irreducible component of the restriction of $E^{\prime}(\mathbf{c})$ to $W_{s}^{0}$; this is a special representation of $W_{s}^{0}$. Hence the induced representation $\operatorname{Ind}_{W_{s}^{0}}^{W}\left(\tilde{E}^{\prime}(\mathbf{c})\right)$ contains a unique irreducible $W$-submodule $E(s, \mathbf{c})$ such that $b_{E(s, \mathbf{c})}=b_{\tilde{E}^{\prime}(\mathbf{c})}$, see $[12,(13.3)]$, 
[9], [11]. It is easy to check that $E(s, \mathbf{c})$ is independent of the choice of $\tilde{E}^{\prime}(\mathbf{c})$, see also $[17,(10.5)]$.

Then there is a well-defined unipotent element $\hat{u}$ in $G$ (up to conjugacy) such that $E(s, \mathbf{c})$ is the Springer representation $E_{\hat{u}, 1}^{G},[12,(13.3)]$. Let $C_{s, \mathbf{c}}$ denote the $G$-conjugacy class of $\hat{u}$. We set $d\left(C_{s, \mathbf{c}}\right):=d(\hat{u})$ (the dimension of the variety $\mathcal{B}_{\hat{u}}^{G}=\mathcal{B}_{\hat{u}}$ of Borel subgroups of $G$ containing $\hat{u}$ ). We have

$$
a_{s, \mathbf{c}}=d\left(C_{s, \mathbf{c}}\right) \text {. }
$$

\section{§3. Character sheaves}

We will assume from now that $p$ is good for $G$. We denote by $G_{\text {unip }}$ the unipotent variety of $G$. By [14, Corollary 11.4] (see also [16, Section 1.6]), there is a surjective map from the set $\hat{G}$ of the character sheaves on $G$ to the $W$-orbits on $T^{*}$. For $s \in T^{*}$, let $\hat{G}_{s}$ be the fibre over $(s)_{G^{*}}$ of this map.

By [14, Corollary 16.7], there exists a well-defined surjective map from $\hat{G}_{s}$ to the set of two-sided cells in $W_{s}$. For $\mathbf{c}$ a two-sided cell in $W_{s}$, we will denote by $\hat{G}_{s, \mathbf{c}}$ the set of character sheaves in the fibre over $\mathbf{c}$ of this map.

Proposition 3.1. Let $s \in T^{*}$ and let $\mathbf{c}$ be a two-sided cell in $W_{s}$, such that, for any $A \in \hat{G}_{s, \mathbf{c}}$, and any $u \in G_{\text {unip }}$ satisfying $A_{\mid\{u\}} \neq 0$, the element $u$ lies in $C_{s, \mathbf{c}}$ or in a unipotent class of dimension strictly smaller than that of $C_{s, \mathbf{c}}$.

Then there exists some $A \in \hat{G}_{s, \mathbf{c}}$ such that $A_{\mid C_{s, \mathbf{c}}} \not \equiv 0$.

Proof. Let us assume that the restriction of each character sheaf $A \in$ $\hat{G}_{s, \mathbf{c}}$ to $C_{s, \mathbf{c}}$ is zero. We choose a Frobenius map $F: G \rightarrow G$ as in section 3.2 below. Then we can similarly associate to the pair $(s, \mathbf{c})$ a set of irreducible representations of $G^{F}$, as in [12]. Let $\pi$ be such a representation. In a similar way as in Lemma 3.3 below, we can assume that $G$ has connected center. Then, using the result of Shoji on Lusztig conjecture [22], we can express the character of $\pi$ as a linear combination of characteristic functions of character sheaves in $\hat{G}_{s, \mathbf{c}}$. The assumption that these character sheaves have zero restriction to $C_{s, \mathbf{c}}$ implies that the restriction of the character $\chi_{\pi}$ of $\pi$ is zero too. Using that expression of the character $\chi_{\pi}$ and the fact that if $A_{\mid C} \not \equiv 0$ for some $A \in \hat{G}_{s, \mathbf{c}}$ and some unipotent class $C$ in $G$ then $\operatorname{dim} C \leq \operatorname{dim} C_{s, \mathbf{c}}$, we obtain that $\chi_{\pi}$ is zero on all unipotent classes of dimension $\geq \operatorname{dim} C_{s, \mathbf{c}}$. Now, the main result of Geck [5, Th. 1.4 (a)] says that there exists a unique $F$-stable unipotent class $C_{\pi}$ in $G$ of maximal 
dimension such that

$$
\sum_{u \in C^{F}} \chi_{\pi}(u) \neq 0 .
$$

It follows that $\operatorname{dim} C_{\pi}<\operatorname{dim} C_{s, \mathbf{c}}$. Moreover, we know by [5, Th. 1.4 (b)] that the $p$-part of the dimension of $\pi$ equals $q^{d\left(C_{\pi}\right)}$, where $d\left(C_{\pi}\right)$ denotes the dimension of the variety of Borel subgroups which contain a fixed element in the class $C_{\pi}$. Using the equality $\operatorname{dim} C_{s, \mathbf{c}}=\operatorname{dim} G-\operatorname{rank}(G)-2 d\left(C_{s, \mathbf{c}}\right)$ (see [24]), we get $d\left(C_{\pi}\right)>d\left(C_{s, \mathbf{c}}\right)$. On the other hand, the $p$-part of the dimension of $\pi$ also equals $q^{a_{s, \mathbf{c}}}$, see $[12,(4.26 .3)]$. It follows that $a_{s, \mathbf{c}}>$ $d\left(C_{s, \mathbf{c}}\right)$, which contradicts the equality (2.2). Hence there exists a character sheaf $A$ in $\hat{G}_{s, \mathbf{c}}$ such that $A_{\mid C_{s, \mathbf{c}}} \not \equiv 0$.

\section{1.}

Lusztig has proved in $[17,(10.9)]$ that, if $p$ is large enough, then the following property always holds.

Property 3.2. For any $s \in T^{*}$, any two-sided cell $\mathbf{c}$ in $W_{s}$, and any $A \in \hat{G}_{s, \mathbf{c}}$, if $C \neq C_{s, \mathbf{c}}$ is a unipotent class in $G$ such that $A_{\mid C} \not \equiv 0$ then $\operatorname{dim} C<\operatorname{dim} C_{s, \mathbf{c}}$.

We remark the following easy fact.

Lemma 3.3. Let $p$ be a prime number. Assume that all reductive connected algebraic groups defined over a finite field of characteristic $p$, which have connected center and are simple modulo their center, satisfy Property 3.2. Then all reductive connected algebraic groups defined over a finite field of characteristic p satisfy Property 3.2.

\section{2 .}

We will assume from now that the center of $G$ is connected. We choose an $\mathbb{F}_{q}$-rational structure on $G$, with corresponding Frobenius map $F: G \rightarrow$ $G$. Replacing $q$ by a power, we assume that $T$ and $T^{*}$ are $F$-stable and split. We choose an element $s \in T^{* F}$, and we fix it from now on. We assume that $F$ acts trivially on $W_{s}$ and $\hat{G}_{s}$. For each irreducible representation $E^{\prime}$ of $W_{s}$ let $R_{s}\left(E^{\prime}\right)$ denote the corresponding almost character, defined in [12, $(3.7)]$ as a certain rational linear combination of Deligne-Lusztig virtual characters of $G^{F}$. For $w \in W$, let $T_{w} \subset G$ denote an $F$-stable maximal torus obtained by twisting the torus $T$ with $w$. Let $\operatorname{Irr}(W)$ denote the set of isomorphism classes of irreducible representations of $W$. Then, using the 
definition of almost characters given in $[12,(3.7)]$, we obtain the following relation:

$$
R_{s}\left(E^{\prime}\right)_{\mid G_{\text {unip }}^{F}}=\left.\sum_{E \in \operatorname{Irr}(W)} m\left(E, E^{\prime}\right) R_{1}(E)\right|_{G_{\text {unip }}^{F}},
$$

where $m\left(E, E^{\prime}\right)$ denotes the multiplicity of $E$ in the representation $\operatorname{Ind}_{W_{s}}^{W}\left(E^{\prime}\right)$.

Let $E$ be an irreducible representation of $W$, and let $(u, \rho)$ be its image under the Springer correspondence $\nu_{T}^{G}$. Replacing $q$ by a power, we assume that $u \in G^{F}$ and that $F$ acts trivially on $A_{G}(u)$. Then (see $[21,(5.2)]$ ), the following holds:

$$
\begin{cases}R_{1}(E)\left(u_{a}\right)=q^{d(u)} \operatorname{trace}(\rho(a)), & \text { for } a \in A_{G}(u) ; \\ R_{1}(E)(g)=0 & \text { if } g \notin \bar{C}_{u}^{F},\end{cases}
$$

where $u_{a}$ are representatives of the $G^{F}$-conjugacy classes in $C^{F}$ associated to $a \in A_{G}(u)$.

\section{3.}

Let $L \supset T$ be a Levi subgroup of a parabolic subgroup $P$ of $G$ and let $\hat{L}$ be the set of character sheaves on $L$. In [14, 4.1], Lusztig introduced the notion of induction $\operatorname{ind}_{L}^{G}$ of character sheaves.

Remark 3.4. Assume that $G$ is simple of adjoint type (with $\operatorname{rank} G \neq$ $0)$. Then $\hat{G}_{s}$ contains at most one cuspidal character sheaf with non-zero restriction to $G_{\text {unip }}$ and such a character sheaf exists exactly in the following cases (see [14, Section 23], [2, (2.3), Appendix A]):

\begin{tabular}{|c|c|c|}
\hline Type of $G$ & Condition on $n$ & Type of $W_{s}$ \\
\hline$B_{n}$ & $n=2 t(t+1)$ & $C_{t(t+1)} \times C_{t(t+1)}$ \\
$C_{n}$ & $n=2 t(4 t \pm 1)$ & $D_{4 t^{2}} \times B_{4 t^{2} \pm 2 t}$ \\
$D_{n}$ & $n=8 t^{2}$ & $D_{4 t^{2}} \times D_{4 t^{2}}$ \\
$G_{2}$ & & $G_{2}$ \\
$F_{4}$ & & $F_{4}$ \\
$E_{8}$ & & $E_{8}$ \\
\hline
\end{tabular}

where $t \geq 1$.

Any character sheaf $A$ of $G$ is obtained as a direct summand of $\operatorname{ind}_{L}^{G}\left(A_{L}\right)$ for the Levi complement $L$ of some parabolic subgroup $P$ of $G$ 
and a cuspidal character sheaf $A_{L}$ on $L$. The pair $\left(L, A_{L}\right)$ is unique up to conjugacy, and we will set

$$
\mathcal{I}(A):=\left\{\left(g L g^{-1}, A_{L}^{g}\right) \mid g \in G\right\}
$$

Assume that the set $\hat{L}^{0}$ of the cuspidal character sheaves on $L$ is nonemtpy. Then by [13, Theorem 9.2 (a)], the group $W_{L}^{G}=\mathrm{N}_{G}(L) / L$ is a finite Coxeter group. For $s \in T^{*}$, we we set $\hat{L}_{s}^{0}:=\hat{L}_{s} \cap \hat{L}^{0}$. Now let $s \in T^{*}$ such that $\hat{L}_{s}^{0}$ is not empty, and let $A_{L} \in \hat{L}_{s}^{0}$. It implies that $s$ is isolated in $L^{*}$, that is, $\mathrm{C}_{L^{*}}(s)$ has the same semisimple rank as $L^{*}$ (see $[14,(17.12)]$ ).

Shoji proves in $[22,(5.16 .1)$ and II, proof of $(4.21)]$ (see also $[12,(8.5)]$ ) that the stabilizer of the cuspidal character sheaf $A_{L}$ in $W_{L}^{G}$ is the image of the canonical map

$$
\left(\mathrm{N}_{G^{*}}\left(L^{*}\right) \cap \mathrm{C}_{G^{*}}(s)\right) / \mathrm{C}_{L^{*}}(s) \hookrightarrow \mathrm{N}_{G^{*}}\left(L^{*}\right) / L^{*},
$$

where $L^{*} \subseteq G^{*}$ denotes the standard Levi subgroup dual to $L$ and $\mathrm{C}_{G^{*}}(s)$ (resp. $\left.\mathrm{C}_{L^{*}}(s)\right)$ denotes the centralizer of $s$ in $G^{*}$ (resp. $L^{*}$ ). We see that this stabilizer only depends on $G, s$ and $L$, and we shall therefore denote it by $W_{L, s}^{G}=W_{L, s}$. Note that we have $W_{T, s}=W_{s}$.

Let $\hat{L}_{\text {unip }}^{0}$ be the subset of all $A_{L} \in \hat{L}^{0}$ such that $A_{L}$ has non-zero

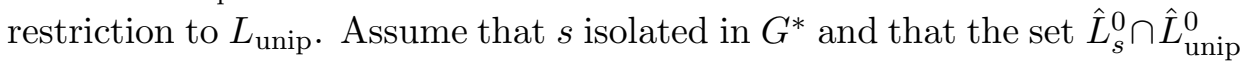
is non-empty.

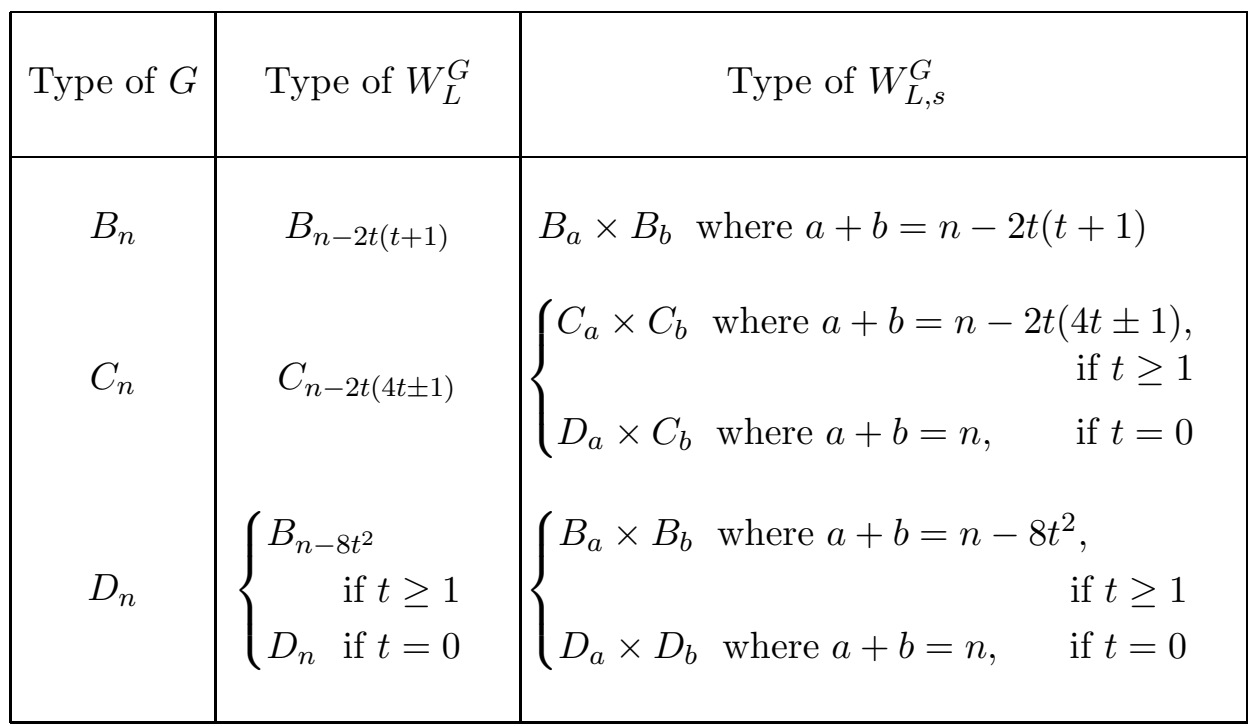


Let $A_{L} \in \hat{L}_{\text {unip }}^{0}$. We set $K:=\operatorname{ind}_{L}^{G}\left(A_{L}\right)$. Now let $\mathcal{H}\left(G, A_{L}\right):=$ $\operatorname{End}_{\mathcal{M} G}(K)$ be the endomorphism algebra of $K$ in $\mathcal{M} G$. It is known by $[15,(2.4)(\mathrm{a}),(2.5)(\mathrm{b})]$ (see also $[13,(3.4)]$ ), that $\mathcal{H}\left(G, A_{L}\right)$ is isomorphic to the group algebra $\overline{\mathbb{Q}}_{\ell}\left[W_{L, s}^{G}\right]$. Hence we have a decomposition

$$
\operatorname{ind}_{L}^{G}\left(A_{L}\right)=\sum_{E^{\prime} \in \operatorname{Irr}\left(W_{L, s}^{G}\right)} E^{\prime} \otimes A_{E^{\prime}}^{s}, \quad \text { where } A_{E^{\prime}}^{s} \in \hat{G}_{s} .
$$

Let $\hat{G}_{\text {unip }}$ be the set of isomorphism classes of characters sheaves $A \in \hat{G}$ such that the restriction of $A$ to $G_{\text {unip }}$ is non-zero. We then get a bijective map from the set $\tilde{\mathcal{T}}(G)$ :

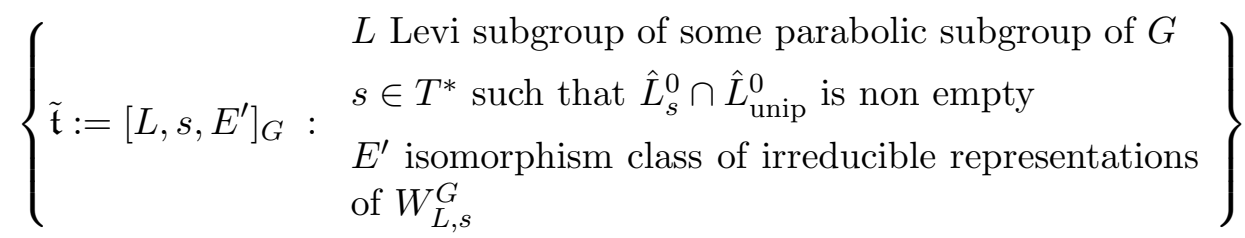

to $\hat{G}_{\text {unip }}$, by sending $\left(L, s, E^{\prime}\right)$ to $A_{E^{\prime}}^{s} \in \hat{G}_{\text {unip }}$. Then $[L, s]_{G}$ will be called the inertial support of $A$. The character sheaves which have non-zero restriction to the unipotent variety of $G$ and have a given inertial support are in bijection with the irreducible characters of the group $W_{L, s}^{G}$. Let $L_{\text {ad }}$ be the adjoint group of $L$ and let pr: $L \rightarrow L_{\text {ad }}$ be the canonical map. Let $L_{\text {der }}$ be the derived subgroup of $L$. By $[14,(17.10)]$, we can write any $A \in \hat{L}^{0}$ in the form $A=\operatorname{pr}^{*}(\bar{A}) \otimes \mathcal{L}$ where $\bar{A} \in \hat{L}_{\text {ad }}^{0}$ and $\mathcal{L}$ is a tame local system on $L$ which is the inverse image of a local system on $L / L_{\text {der }}$ under the canonical map $L \rightarrow L / L_{\text {der }}$.

We have a corresponding embedding of dual groups $L_{\text {der }}^{*} \subseteq L^{*}$. If $\bar{A}_{L}$ lies in the series of $L_{\text {ad }}$ defined by $\bar{s} \in T^{*} \cap L_{\text {der }}^{*}$ and $\mathcal{L}$ corresponds to the central element $z$ of $L^{*}$, then $A_{L}$ lies in the series of $L$ defined by $s:=\bar{s} z$. Clearly, we have $\mathrm{C}_{L^{*}}(s)=\mathrm{C}_{L^{*}}(\bar{s})$. Note that if $L=T$ then $\bar{s}=1$.

Assume that $L_{\text {ad }}$ has a cuspidal character sheaf $\bar{A}_{L}$ such that $\bar{A}_{L}$ has non-zero restriction to the unipotent variety of $L_{\mathrm{ad}}$. Then $\bar{A}_{L}$ is uniquely determined. Let $\bar{s} \in T^{*} \cap L_{\mathrm{der}}^{*} \subseteq L^{*}$ such that $\bar{A}_{L}$ lies in the series defined by $\bar{s}$. By $[13$, Theorem $9.2(\mathrm{~b})]$, we have $W_{L, \bar{s}}^{G}=W_{L}^{G}$. We consider the decomposition

$$
\operatorname{ind}_{L}^{G}\left(\operatorname{pr}^{*}\left(\bar{A}_{L}\right)\right)=\sum_{E \in \operatorname{Irr}\left(W_{L}^{G}\right)} E \otimes A_{E}^{\bar{s}}, \quad \text { where } A_{E}^{\bar{s}} \in \hat{G}_{\bar{s}} .
$$


Let $A_{L} \in \hat{L}$ be any cuspidal character sheaf. If the restriction of $A_{L}$ to $L_{\text {unip }}$ is zero then the restrictions of all components of $\operatorname{ind}_{L}^{G}\left(A_{L}\right)$ to $G_{\text {unip }}$ will also be zero (see $[15,(2.9)]$ ). Assume now that the restriction of $A_{L}$ to $L_{\text {unip }}$ is non-zero. We can write $A_{L}=\operatorname{pr}^{*}\left(\bar{A}_{L}\right) \otimes \mathcal{L}$ where $\bar{A}_{L}$ lies in the series of $L_{\text {ad }}$ defined by $\bar{s} \in T * \cap L *$ der and $\mathcal{L}$ is pulled back from a local system on $L / L_{\mathrm{der}}$. Let $A_{L}, \bar{A}_{L}$ lie in the series defined by $s, \bar{s} \in T^{*}$, respectively.

It is clear that $A_{L}$ and $\operatorname{pr}^{*}\left(\bar{A}_{L}\right)$ have the same restriction to $L_{\text {unip }},[15$, (2.6) (c)]. Moreover, the restriction of the decomposition to $G_{\text {unip }}$ is related to the restriction of that in (a) by the following formula, see $[15,(2.6)(\mathrm{e})]$ :

$$
A_{E^{\prime}}^{s}=\bigoplus_{E} m\left(E, E^{\prime}\right) A_{E}^{\bar{s}} \text { on } G_{\text {unip }}
$$

where $E^{\prime} \in \operatorname{Irr}\left(W_{L, s}^{G}\right), E \in \operatorname{Irr}\left(W_{L}^{G}\right)$ and $m\left(E, E^{\prime}\right)$ denotes the multiplicity of $E$ in the induced representation $\operatorname{Ind}_{W_{L, s}^{G}}^{W_{G}^{G}}\left(E^{\prime}\right)$.

Proposition 3.5. Let $E^{\prime}$ be an irreducible representation of $W_{T, s}=$ $W_{s}$, and let $\mathbf{c}$ be the two-sided cell in $W_{s}$ to which $E^{\prime}$ belongs. If $C$ is a unipotent class in $G$ such that $A_{E^{\prime}}^{s} \mid C \not \equiv 0$, then $\operatorname{dim} C \leq \operatorname{dim} C_{s, \mathbf{c}}$, with equality only for $C=C_{s, \mathbf{c}}$.

Proof. Assume first that $s=1$. Then $W_{s}=W$. We can choose a Frobenius map $F: G \rightarrow G$ as in (3.2) so that the characteristic function of $A_{E^{\prime}}^{1}$ coincides (for a suitable normalization) with the almost character $R_{1}\left(E^{\prime}\right)$ of $G^{F}$, see, for instance, [7, Cor. 2.3.2]. We have seen that, via the Springer correspondence, the representation $E^{\prime}$ corresponds to a pair $\left(C^{\prime}, \mathcal{E}^{\prime}\right)$, where $C^{\prime}$ is a unipotent class in $G$ and $\mathcal{E}^{\prime}$ is a certain $G$-invariant irreducible local system on $C^{\prime}$, and that the restriction of the almost character $R_{1}\left(E^{\prime}\right)$ to $G_{\text {unip }}^{F}$ has non-zero values only in $\bar{C}^{\prime F}$ (see $(3.2)$ ). The unique special character belonging to $\mathbf{c}$ corresponds to the pair $\left(C_{s, \mathbf{c}}, \overline{\mathbb{Q}}_{\ell}\right)$ via the Springer correspondence, and we have seen that $a_{s, \mathbf{c}}=d\left(C_{s, \mathbf{c}}\right)$. By [17, Cor. 10.9], we have $\operatorname{dim} C^{\prime} \leq \operatorname{dim} C_{s, \mathbf{c}}$, with equality only for $C^{\prime}=C_{s, \mathbf{c}}$. Note that this statement is true whenever $p$ is good for $G$, since the Springer correspondence as well the dimensions of varieties of Borel subgroups are independent of the characteristic as long as the characteristic is good (see the tables in [3], for instance). Now, if $C$ is a unipotent class such that $A_{E^{\prime} \mid\{u\}}^{s} \neq 0$ for some $u \in C$, then we may assume, as in $(3.2)$, that $F(u)=u$ 
and that $R_{1}\left(E^{\prime}\right)(u) \neq 0$, and the above remarks on the values of Green functions imply that the class $C$ lies in the Zariski closure of $C^{\prime}$. Hence we have $\operatorname{dim} C \leq \operatorname{dim} C^{\prime} \leq \operatorname{dim} C_{s, \mathbf{c}}$, with equality only for $C=C^{\prime}=C_{s, \mathbf{c}}$.

When $s$ is not equal to 1 , the result follows from [17, Cor. 10.9], the formula (3.1), and the case $s=1$.

We are now able to prove Theorem 1.1 for groups of exceptional type.

Proposition 3.6. Assume that $G$ is of exceptional type. Then Theorem 1.1 holds for $G$ when $p$ is good.

Proof. By using Lemma 3.3, we can assume that $G$ is simple modulo its center, which can be assumed to be connected. Then let $A \in \hat{G}$, and let $\left(L, A_{L}\right) \in \mathcal{I}(A)$. Since $G$ is of exceptional type, the only possibilities for $L$ are either $L=T$ or $L=G$, see [14, §18]. When $L=T$, the result follows from Proposition 3.5. When $L=G$, we have $A=\mathrm{IC}\left(\bar{C} \cdot \mathrm{Z}_{G}, \mathcal{E} \otimes \mathcal{L}\right)[\operatorname{dim}(\bar{C}$. $\left.\mathrm{Z}_{G}\right)$ ], where $(C, \mathcal{E})$ is a cuspidal pair, and $\mathcal{L}$ is a tame local system on $L$. If $G$ is of type $E_{6}, E_{7}$, there are no cuspidal pairs for $L=G$ which are supported on a unipotent class, see [2, Appendix].

Hence we can assume that $G$ is of type $G_{2}, F_{4}, E_{8}$. Let $C^{\prime}$ be a unipotent class in $G$ such that $A_{\mid C^{\prime}} \not \equiv 0$. It implies that $C^{\prime}$ is contained in the Zariski closure of $C$. The unique special representation $E$ of $W$ belonging to $\mathbf{c}$ corresponds to the pair $\left(C_{s, \mathbf{c}}, \overline{\mathbb{Q}}_{\ell}\right)$ via the Springer correspondence. By [14, (20.6) and $\S 21]$, we know that $E$ belongs to the unique family $\mathcal{F}$ of representations of $W$ with 4, 7, 17 elements, for $G$ of type $G_{2}, F_{4}, E_{8}$, respectively. But, by $[12,(13.1 .3)]$, the order of $A_{G}(u)$ for $u \in C_{s, \mathbf{c}}$ equals the order of the finite group $\mathcal{G}_{\mathcal{F}}$ associated to the family $\mathcal{F}$. Hence the order of $A_{G}(u)$ is $6,24,120$, for $G$ of type $G_{2}, F_{4}, E_{8}$, respectively. But, for $G$ of type $G_{2}, F_{4}, E_{8}$, there is exactly one cuspidal pair for $L=G$ supported on a unipotent class, say $C$, and the class $C$ is uniquely determined by the condition that the group $A_{G}(u)$, for $u \in C$, has order $6,24,120$, respectively. Hence $C=C_{s, \mathbf{c}}$. The assertion (a) of Theorem 1.1 follows. Then the assertion (b) follows from Proposition 3.1.

Remark 3.7. The case of groups of classical type is more difficult, because it involves the group $W_{L, s}$, with $L \neq T$, and not only the group $W_{s}$. Because of that we will need to use the generalized Springer Correspondence instead of the ordinary one. In particular, we will prove a generalization of [17, Cor. 10.9] in that case. 


\section{$\S 4$. The classical groups case}

In this section, we will prove Theorem 1.1 for classical groups under the assumption that $p$ is odd.

\subsection{The strategy of the proof}

First note that, by Proposition 3.1, it is sufficient to prove the assertion (a) of Theorem 1.1. Note also that if $L=T$, it follows from Proposition 3.5. Hence we can, if necessary, assume that $L \neq T$ (we will assume this when $G$ is of type $C$ or $D$ ). By Lemma 3.3, we can and do assume that the center of $G$ is connected and that $G$ is simple modulo its center.

We will first show that the proof can be reduced to the case where $s \in G^{*}$ is isolated semisimple: by using a similar argument as in the end of the proof of Proposition 3.5, the formula 3.7 shows that in order to investigate the restriction of $A_{E^{\prime}}^{s}$ to the unipotent variety $G_{\text {unip }}$, it is enough to consider the case where the cuspidal character sheaf $A_{L}$ has support on $G_{\text {unip }}$. But in the case of classical groups, $A_{L}$ satisfies this condition only when $A_{L} \in \hat{L}_{s}$ with $s$ of order 2 (see [14, (17.12) and §23] or [18]), in which case $s$ is isolated. From now on we will assume that $s$ is isolated. Our proof will be based on the generalized Springer correspondence.

\subsubsection{The generalized Springer correspondence}

Let $\mathfrak{B}(G)$ denote the set of $G$-conjugacy classes of triples $\left(L, u_{L}, \rho_{L}\right)$ where $L$ is the Levi subgroup of some parabolic subgroup of $G, u_{L}$ is a unipotent element in $L$ and $\rho_{L}$ is an irreducible representation of the group $A_{L}\left(u_{L}\right)$ such that the pair $\left(u_{L}, \rho_{L}\right)$ is cuspidal in $L$. We denote by $\left[L, u_{L}, \rho_{L}\right]_{G}$ the $G$-conjugacy class of $\left(L, u_{L}, \rho_{L}\right)$. We set $W_{L}^{G}:=\mathrm{N}_{G}(L) / L$ (it is a Coxeter group, see [13, Th. 9.2]), and we denote by $\operatorname{Irr}\left(W_{L}^{G}\right)$ the set of all the isomorphism classes of irreducible representations of the group $W_{L}^{G}$. Let

$$
\nu^{G}: \bigsqcup_{\substack{\mathfrak{b} \in \mathfrak{B}(G) \\ \mathfrak{b}=\left[L, u_{L}, \rho_{L}\right]_{G}}} \operatorname{Irr}\left(W_{L}^{G}\right) \longrightarrow \mathcal{N}_{G}
$$

be the generalized Springer correspondence $[13, \S 6]$.

Let $\mathfrak{n}=(C, \mathcal{E}) \in \mathcal{N}_{G}$. We choose the natural mixed structure on $\mathcal{E}$, that is, the one (see $[15, \oint 3.2-3.4]$ ) which has the property that $F^{*} \mathcal{E} \stackrel{\sim}{\rightarrow} \mathcal{E}$ induces on the stalk over a split element $u$ of $C$ the identity map times $q^{\frac{1}{2}\left(\operatorname{dim} G-d_{\mathfrak{n}}\right)}$. (This property characterizes the mixed structure on $\mathcal{E}$.) This 
mixed structure on $A_{L}$ extending the natural mixed structure on $\mathcal{E}_{L}$ is compatible with the isomorphism $\mathcal{H}\left(G, A_{L}\right) \simeq \overline{\mathbb{Q}}_{\ell}\left[W_{L, s}\right]$. The induced complex $\operatorname{ind}_{L}^{G}\left(A_{L}\right)$ inherits a natural mixed structure, and we will denote by $\chi_{A_{E^{\prime}}^{s}}$ the corresponding characteristic function of $A_{E^{\prime}}^{s}$.

On the other hand, we associate with any $(C, \mathcal{E}) \in \mathcal{N}_{G}$ the class function $Y_{(C, \mathcal{E})}: G^{F} \rightarrow \overline{\mathbb{Q}}_{\ell}$ defined as follows:

$$
Y_{(C, \mathcal{E})}(g):=\chi_{\mathrm{IC}(\bar{C}, \mathcal{E})}(g), \quad g \in \bar{C}^{F},
$$

extended by zero on $G^{F}-\bar{C}^{F}$ (where the mixed structure on $\operatorname{IC}(\bar{C}, \mathcal{E})$ is that extending the natural one on $\mathcal{E}$ ).

We assume that $G$ is a split classical group of adjoint type and that $p$ is odd. Let $s \in T^{*}$ and $E^{\prime} \in \operatorname{Irr}\left(W_{L, s}\right)$. If $L^{*} \supset T^{*}$ is a dual pair to $L \supset T$, we will set

$$
W_{L, s}^{G}=W_{L, s}:=\mathrm{N}_{\mathrm{C}_{G^{*}(s)}}\left(\mathrm{C}_{L^{*}}^{0}(s)\right) / \mathrm{C}_{L^{*}}^{0}(s) .
$$

Let $C$ be an $F$-stable unipotent class in $G$ and let $u \in C^{F}$ be a split element. If $s$ is isolated in $G^{*}$ and $\mathrm{C}_{L^{*}}(s)$ is cuspidal, then (see [2, Th. 3.2]) we have the following formula

$$
\chi_{A_{E^{\prime}}^{s}}(u)=\sum_{E \in \operatorname{Irr}\left(W_{L}^{G}\right)} m\left(E, E^{\prime}\right)(-1)^{\operatorname{rank} G} q^{\operatorname{dim} G-d\left(\nu^{G}(E)\right)} Y_{\nu^{G}(E)}(u),
$$

where $m\left(E, E^{\prime}\right)$ denotes the multiplicity of $E$ in the representation $\operatorname{Ind}_{W_{L, s}^{G}}^{W_{L}^{G}}\left(E^{\prime}\right)$.

4.1.2. Scheme of the proof of assertion (a) of Theorem 1.1

Following Lusztig (see [13]), we will consider a set $\Psi^{G}$ and a map

$$
\psi^{G}: \Psi^{G} \longrightarrow \mathcal{N}_{G}
$$

which is essentially a bijection such that

- $\Psi^{G}$ is the disjoint union of subsets $\Psi_{L}^{G}$ where $L \supset T$ are cuspidal Levi subgroups of $G$,

- there exists a set $\Psi_{T}^{H}$, an injective map $\psi^{H}: \Psi_{T}^{H} \hookrightarrow \mathcal{N}_{H}$, and two bijections

$$
\Delta_{L}^{G}: \Psi_{T}^{H} \longrightarrow \Psi_{L}^{G} \text { and } \Theta_{L}^{G}: \operatorname{Irr}\left(W_{L}^{G}\right) \longrightarrow \Psi_{T}^{H},
$$

for $H$ a reductive group with Weyl group $W^{H}=W_{L}^{G}$, satisfying, for every $E \in \operatorname{Irr}\left(W_{L}^{G}\right)$, 


$$
\nu_{T}^{H}(E)=\left(\psi^{H} \circ \Theta_{L}^{G}\right)(E),
$$

where $\nu_{T}^{H}: \operatorname{Irr}\left(W^{H}\right) \hookrightarrow \mathcal{N}_{H}$ is the (ordinary) Springer correspondence, and

$$
\nu^{G}(E)=\left(\psi^{G} \circ \Delta_{L}^{G} \circ \Theta_{L}^{G}\right)(E) .
$$

For $(C, \mathcal{E}) \in \mathcal{N}_{G}$, we will set $\Pi(C, \mathcal{E}):=C$. Let $E^{\prime} \in \operatorname{Irr}\left(W_{L, s}^{G}\right)$ and let $\mathbf{c}$ be the two-sided cell of $W_{s}$ such that $A_{E^{\prime}}^{s}$ belongs to $\hat{G}_{s, \mathbf{c}}$. Assuming that the element $s$ is isolated in $G^{*}$, we will prove that there exists $E_{\max } \in \operatorname{Irr}\left(W_{L}^{G}\right)$ occurring with multiplicity one in the induced representation $\operatorname{Ind}_{W_{L, s}^{G}}^{W_{G}^{G}}\left(E^{\prime}\right)$ such that the following hold

(i) for any $E \in \operatorname{Irr}\left(W_{L}^{G}\right)$ occurring in $\operatorname{Ind}_{W_{L, s}^{G}}^{W^{G}}\left(E^{\prime}\right)$ : $\operatorname{dim} \Pi\left(\nu^{G}(E)\right) \leq \operatorname{dim} \Pi\left(\nu^{G}\left(E_{\max }\right)\right)$, with equality only for $E=E_{\max }$.

We set $C_{A}:=\Pi\left(\nu^{G}\left(E_{\max }\right)\right)$, for $A=A_{E^{\prime}}^{s}$. Then it follows from 4.4, using (i), that

(*) the restriction of $A$ to $C_{A}$ does not vanish, and that $\operatorname{dim} C<\operatorname{dim} C_{A}$, for any unipotent class $C \neq C_{A}$ in $G$ such that the restriction of $A$ to $C$ does not vanish.

We will now prove that $C_{A}=C_{s, \mathbf{c}}$, for some $A \in \hat{G}_{s, \mathbf{c}}$. We first assume that $p$ is large enough so that Lusztig's result [17, Th. 10.7] is applicable. Let $s \in T^{*}$ and let $\mathbf{c}$ be a two-sided cell in $W_{s}$. By [17, Th. 10.7 (ii)], we see that Proposition 3.1 is applicable. It follows that there exists $A \in \hat{G}_{s, \mathbf{c}}$ such that $\left.A\right|_{C_{s, \mathbf{c}}} \not \equiv 0$. Assume that $s$ is isolated in order to be able to apply (i). Then using $(*)$ we get $\operatorname{dim} C_{s, \mathbf{c}} \leq \operatorname{dim} C_{A}$. Conversely, since we have seen that $A$ does not vanish on $C_{A}$, by applying [17, Th. 10.7] again, we obtain $\operatorname{dim} C_{A} \leq \operatorname{dim} C_{s, \mathbf{c}}$. Hence $\operatorname{dim} C_{A}=\operatorname{dim} C_{s, \mathbf{c}}$. Then by using (*), we get $C_{s, \mathbf{c}}=C_{A}$, when $p$ is sufficiently large.

Now we assume only that $p$ is good for $G$. Let $q^{\prime}$ be a power of a large prime and let $G^{\prime}$ be a group defined over $\mathbb{F}_{q^{\prime}}$ of the same type as $G$, with the same Weyl group $W$. We can choose $q^{\prime}$ in such a way that there exists a semisimple element $s^{\prime}$ in the dual group of $G^{\prime}$ such that $W_{s^{\prime}}^{G^{\prime}} \subset W$ can be identified with $W_{s}$. We can assume that $q^{\prime}$ was chosen 
large enough such that the results in [17] are applicable. Since $p$ is good for $G$, it follows from [24, Th. III 5.2], from the explicit results obtained by Springer, Hotta-Springer, Shoji and Alvis-Lusztig (see [25], [6], [19], [20], [1]) and from the link between the generalized Springer correspondence for $G$ and the (ordinary) Springer correspondence for $H$ (see (4.5), (4.6)) that there exists an isomorphism $\eta_{G}$ of partially ordered sets from the set of unipotent classes in $G^{\prime}$ to the set of unipotent classes in $G$ such that

$$
\eta_{G}\left(\Pi\left(\nu^{G^{\prime}}(E)\right)\right)=\Pi\left(\nu^{G}(E)\right), \quad \text { for every } E \in \operatorname{Irr}\left(W_{L}^{G}\right) .
$$

Hence $\eta_{G}\left(\Pi\left(\nu^{G^{\prime}}\left(E_{\max }\right)\right)\right)=\Pi\left(\nu^{G}\left(E_{\max }\right)\right)$. Since $\eta_{G}\left(C_{s^{\prime}, \mathbf{c}}\right)=C_{s, \mathbf{c}}$ (because the class $C_{s, \mathbf{c}}$ is defined using the Springer correspondence), and since $\Pi\left(\nu^{G^{\prime}}\left(E_{\max }\right)\right)=C_{s^{\prime}, \mathbf{c}}$, we finally obtain $C_{A}=C_{s, \mathbf{c}}$.

The strategy of proving (i) is as follows. We will consider a bijection $\Sigma_{L}^{G}$ from $\operatorname{Irr}\left(W_{L}^{G}\right)$ to a certain partially ordered set $\Phi_{T}^{H}$ (with partial order denoted by $\left.\underset{\Phi_{T}^{H}}{\leq}\right)$, and show that

$(* *)$ there exists $E_{\max } \in \operatorname{Irr}\left(W_{L}^{G}\right)$ occurring with mutiplicity one in $\operatorname{Ind}_{W_{L, s}^{G}}^{W_{L}^{G}}\left(E^{\prime}\right)$ such that

$$
\Sigma_{L}^{G}(E) \underset{\Phi_{T}^{H}}{\leq \Sigma_{L}^{G}}\left(E_{\max }\right), \quad \text { for every } E \text { occurring in } \operatorname{Ind}_{W_{L, s}^{G}}^{W_{L}^{G}}\left(E^{\prime}\right) .
$$

The statement (**) will follow easily from Corollary 4.3 and Proposition 4.1 (the latter studying the case of groups of type A).

On the other hand, we will set

$$
\sigma_{L}^{G}:=\Theta_{L}^{G} \circ\left(\Sigma_{L}^{G}\right)^{-1}
$$

$\sigma_{L}^{G}$ is a bijection from $\Phi_{T}^{H}$ to $\Psi_{T}^{H}$. In (4.3.1), we will consider a naive order on $\Psi_{T}^{H}$ (resp. $\left.\Psi_{L}^{G}\right)$, denoted by $\underset{\Psi_{T}^{H}}{\leq}\left(\operatorname{resp} \underset{\Psi_{L}^{G}}{\leq}\right)$ such that, for any $\Lambda_{T}$, $\tilde{\Lambda}_{T}$ in $\Phi_{T}^{H}$, we have $\Lambda_{T} \underset{\Phi_{T}^{H}}{\leq} \tilde{\Lambda}_{T}$ if and only if $\sigma_{L}^{G}\left(\Lambda_{T}\right) \underset{\Psi_{T}^{H}}{\leq} \sigma_{L}^{G}\left(\tilde{\Lambda}_{T}\right)\left(\operatorname{resp} .\left(\Delta_{L}^{G} \circ\right.\right.$ $\left.\sigma_{L}^{G}\right)\left(\Lambda_{T}\right) \underset{\Psi_{L}^{G}}{\leq}\left(\Delta_{L}^{G} \circ \sigma_{L}^{G}\right)\left(\tilde{\Lambda}_{T}\right)$. Finally, we will prove in Proposition 4.4 that if $\theta$, $\tilde{\theta} \in \Psi_{L}^{G}$ satisfy $\theta \underset{\Psi_{L}^{G}}{\leq} \tilde{\theta}$, then $\operatorname{dim} C(\theta) \leq \operatorname{dim} C(\tilde{\theta})$, where $C(\theta):=\Pi\left(\psi^{G}(\theta)\right)$, $C(\tilde{\theta}):=\Pi\left(\psi^{G}(\tilde{\theta})\right)$ and that the function $\Psi_{L}^{G} \ni \theta \mapsto \operatorname{dim} \Pi\left(\psi^{G}(\theta)\right)$ attains it maximum at a unique element of $\Psi_{L}^{G}$. Then by applying (**) we will get (i). 


\subsubsection{Type of the Levi subgroup}

First, we note that by the explicit classification of cuspidal character sheaves recalled in (3.4) the cuspidal Levi subgroups $L$ must be of type isomorphic to

$$
\begin{array}{ll}
\left(B_{n}\right): & \mathrm{SO}_{(2 t+1)^{2}} \times \mathrm{GL}_{1} \times \cdots \times \mathrm{GL}_{1}, \\
\left(C_{n}\right): & \mathrm{PSp}_{16 t^{2} \pm 4 t} \times \mathrm{GL}_{1} \times \cdots \times \mathrm{GL}_{1}, \\
\left(D_{n}\right): & \mathrm{PSO}_{16 t^{2}} \times \mathrm{GL}_{1} \times \cdots \times \mathrm{GL}_{1},
\end{array}
$$

for some integer $t \geq 0$. We denote by $\tilde{n}$ the number of factors $\mathrm{GL}_{1}$ in each type of Levi above, that is:

$$
\begin{array}{ll}
\left(B_{n}\right): & \tilde{n}=n-2 t^{2}-2 t, \\
\left(C_{n}\right): & \tilde{n}=n-\left(8 t^{2} \pm 2 t\right), \\
\left(D_{n}\right): & \tilde{n}=n-8 t^{2} .
\end{array}
$$

\subsubsection{Description of the ramification subgroup of $L$}

We denote by $W_{n}$ the group of all permutations of the set $\{1,2, \ldots, n$, $\left.n^{*}, \ldots, 2^{*}, 1^{*}\right\}$ which commute with the involution $i \mapsto i^{*}, i^{*} \mapsto i,(1 \leq$ $i \leq n)$. We set $W_{0}=\{1\}$. For each $j, 1 \leq j \leq n-1$, let $s_{j} \in W_{n}$ be the permutation which interchanges $j$ with $j+1$ and also $j^{*}$ with $j^{*}+1$ and leaves the other elements unchanged. Let $\sigma_{a} \in W_{n}(1 \leq a \leq n)$ be the permutation which interchanges $a$ with $a^{*}$ and leaves the other elements unchanged. Let $S=\left\{s_{1}, s_{2}, \ldots, s_{n-1}, \sigma_{n}\right\}$. Then $\left(W_{n}, S\right)$ is a Coxeter group of type $B_{n}=C_{n}$.

Let $\varphi=\varphi_{n}: W_{n} \rightarrow\{-1,1\}$ be the homorphism defined by the condition $\varphi_{n}\left(s_{j}\right)=1(1 \leq j \leq n-1), \varphi_{n}\left(\sigma_{n}\right)=-1$. Let $W_{n}^{\prime}$ be the kernel of $\varphi$. It is a Coxeter group of type $D_{n}$.

Then we can identify the group $W_{L}^{G}$ with the group $W_{\tilde{n}}$ if $G$ is of type $B_{n}$ or $C_{n}$, or if $t \geq 1$ and $G$ is of type $D_{n}$ as follows. Consider a basis $e_{1}, \ldots$, $e_{n}, e_{n}^{*}, \ldots, e_{1}^{*}$ of $V$ such that $\left(e_{i}, e_{i}^{*}\right)=1,\left(e_{i}^{*}, e_{i}\right)=1\left(\operatorname{resp} .\left(e_{i}^{*}, e_{i}\right)=-1\right)$ if $(,$,$) is orthogonal (resp. symplectic) and all other scalar products equal to$ zero. We assume that $L$ is the set of $g \in G$ which map each of the vectors $e_{1}, \ldots, e_{\tilde{n}}, e_{\tilde{n}}^{*}, \ldots, e_{1}^{*}$ into a scalar multiple of itself. Then each element of $\mathrm{N}_{G}(L) / L$ defines a permutation of the set of lines $\left\langle e_{1}\right\rangle, \ldots,\left\langle e_{\tilde{n}}\right\rangle,\left\langle e_{\tilde{n}}^{*}\right\rangle, \ldots$, $\left\langle e_{1}^{*}\right\rangle$ and this gives the wanted isomorphism. If $G$ is of type $D_{n}$ and $t=0$ then $W_{L}^{G}=W_{\tilde{n}}^{\prime}=W_{n}^{\prime}$. 
4.1.5. Dual side

The group $L^{*}$ is of type

$\left(B_{n}\right): \quad \mathrm{Sp}_{4 t^{2}+4 t} \times \mathrm{GL}_{1} \times \cdots \times \mathrm{GL}_{1}$,

$\left(C_{n}\right): \quad \operatorname{Spin}_{16 t^{2} \pm 4 t+1} \times \mathrm{GL}_{1} \times \cdots \times \mathrm{GL}_{1}$,

$\left(D_{n}\right): \quad \operatorname{Spin}_{16 t^{2}} \times \mathrm{GL}_{1} \times \cdots \times \mathrm{GL}_{1}$.

Since the cuspidal character sheaf $A$ of $L$ is supported by the closure of a unipotent class, the group $\mathrm{C}_{L^{*}}(s)$ is of type

$\left(B_{n}\right): \quad \mathrm{Sp}_{2 t^{2}+2 t} \times \mathrm{Sp}_{2 t^{2}+2 t} \times \mathrm{GL}_{1} \times \cdots \times \mathrm{GL}_{1}$,

$\left(C_{n}\right): \quad \operatorname{Spin}_{8 t^{2}} \times \operatorname{Spin}_{8 t^{2} \pm 4 t+1} \times \mathrm{GL}_{1} \times \cdots \times \mathrm{GL}_{1}$,

$\left(D_{n}\right): \quad \operatorname{Spin}_{8 t^{2}} \times \operatorname{Spin}_{8 t^{2}} \times \mathrm{GL}_{1} \times \cdots \times \mathrm{GL}_{1}$.

4.1.6. The ramification subgroup of the cuspidal pair

We can identify in a standard way the group $W_{L, s}^{G}$ with

- $W_{\tilde{n}^{\prime}} \times W_{\tilde{n}^{\prime \prime}}$ if $G$ is of type $B_{n}$ or if $t \geq 1$ and $G$ is of type $C_{n}$ or $D_{n}$,

- $W_{\tilde{n}^{\prime}}^{\prime} \times W_{\tilde{n}^{\prime \prime}}$ if $G$ is of type $C_{n}$ and $t=0$,

- $W_{\tilde{n}^{\prime}}^{\prime} \times W_{\tilde{n}^{\prime \prime}}^{\prime}$ if $G$ is of type $D_{n}$ and $t=0$

where $\tilde{n}^{\prime}+\tilde{n}^{\prime \prime}=\tilde{n}$; the isomorphism is canonical up to conjugation with inner automorphism of $W_{\tilde{n}^{\prime}} \times W_{\tilde{n}^{\prime \prime}}\left(\right.$ resp. $\left.W_{\tilde{n}^{\prime}}^{\prime} \times W_{\tilde{n}^{\prime \prime}}, W_{\tilde{n}^{\prime}}^{\prime} \times W_{\tilde{n}^{\prime \prime}}^{\prime}\right)$. Hence an irreducible representation of $W_{L, s}^{G}$ may be identified with the corresponding representation of $W_{\tilde{n}^{\prime}} \times W_{\tilde{n}^{\prime \prime}}\left(\right.$ resp. $\left.W_{\tilde{n}^{\prime}}^{\prime} \times W_{\tilde{n}^{\prime \prime}}, W_{\tilde{n}^{\prime}}^{\prime} \times W_{\tilde{n}^{\prime \prime}}^{\prime}\right)$.

4.1.7. The associated combinatorial data

For $L$ and $\tilde{n}$ are as in (4.1.3), we set

- $d:=1 \pm 4 t, \Psi_{L}^{G}:=\Psi_{2 n, d}, H:=\operatorname{PSp}_{2 \tilde{n}}, \Psi_{T}^{H}:=\Psi_{2 \tilde{n}, 1}$, when $G=\operatorname{PSp}_{2 n}$;

- $d:=2 t+1, \Psi_{L}^{G}:=\Psi_{2 n+1, d}^{\prime}, H:=\mathrm{SO}_{2 \tilde{n}+1}, \Psi_{T}^{H}:=\Psi_{2 \tilde{n}+1,1}^{\prime}$, when $G=\mathrm{SO}_{2 n+1}$

- $d:=4 t, \Psi_{L}^{G}:=\Psi_{2 n+1, d}^{\prime}, H:=\mathrm{SO}_{2 \tilde{n}+1}, \Psi_{T}^{H}:=\Psi_{2 \tilde{n}+1,1}^{\prime}$, when $G=$ $\mathrm{SO}_{2 n}$ and $t \geq 1$

- $\Psi_{L}^{G}:=\Psi_{N}^{\prime 0}=\Psi_{T}^{H}, H:=G$, when $G=\mathrm{SO}_{2 n}$ and $t=0$. 


\subsection{On induced representations of Weyl groups of classical type}

4.2.1.

Let $\mathfrak{S}_{\tilde{n}}$ be the permutation group of the set $\{1,2, \ldots, \tilde{n}\}$. We write $\alpha \dashv \tilde{n}$ to mean that $\alpha$ is a partition of the integer $\tilde{n}$. Each $E \in \operatorname{Irr}\left(\mathfrak{S}_{\tilde{n}}\right)$ is parametrized by a partition of $\tilde{n}$; we shall denote by $E_{\alpha}$ the irreducible representation of $\mathfrak{S}_{\tilde{n}}$ corresponding to the partition $\alpha$.

Let $\tilde{n}^{\prime}, \tilde{n}^{\prime \prime}$ be two non-negative integers such that $\tilde{n}^{\prime}+\tilde{n}^{\prime \prime}=\tilde{n}$. We construct from representations $E_{\alpha^{\prime}}$ of $\mathfrak{S}_{\tilde{n}^{\prime}}$ and $E_{\alpha^{\prime \prime}}$ of $\mathfrak{S}_{\tilde{n}^{\prime \prime}}$ the representation

$$
I\left(\alpha^{\prime}, \alpha^{\prime \prime}\right):=\operatorname{Ind}_{\mathfrak{S}_{\tilde{n}^{\prime}} \times \mathfrak{S}_{\tilde{n}^{\prime \prime}}}^{\mathfrak{S}_{\tilde{n}}}\left(E_{\alpha^{\prime}} \otimes E_{\alpha^{\prime \prime}}\right)
$$

of $\mathfrak{S}_{\tilde{n}}$ induced from the tensor product representation $E_{\alpha^{\prime}} \otimes E_{\alpha^{\prime \prime}}$ of the subgroup $\mathfrak{S}_{\tilde{n}^{\prime}} \times \mathfrak{S}_{\tilde{n}^{\prime \prime}}$ of $\mathfrak{S}_{\tilde{n}}$. Let

$$
I\left(\alpha^{\prime}, \alpha^{\prime \prime}\right)=\sum_{\alpha \dashv n} m\left(\alpha^{\prime \prime}, \alpha \backslash \alpha^{\prime}\right) E_{\alpha}
$$

be the irreducible decomposition.

Let $\leq$ be the natural partial order on partitions defined as follows. Let $\alpha=\left(0 \leq \alpha_{0} \leq \alpha_{1} \leq \cdots \leq \alpha_{m}\right)$ and $\beta=\left(0 \leq \beta_{0} \leq \beta_{1} \leq \cdots \leq \beta_{m}\right)$ be two partitions of $\tilde{n}$. Then

$$
\begin{gathered}
\beta \leq \alpha \quad \text { if } \begin{aligned}
\beta_{m} & \leq \alpha_{m} \\
\beta_{m-1}+\beta_{m} & \leq \alpha_{m-1}+\alpha_{m} \\
& \vdots \\
\beta_{m-2}+\beta_{m-1}+\beta_{m} & \leq \alpha_{m-2}+\alpha_{m-1}+\alpha_{m} \\
& \vdots \\
\beta_{1}+\beta_{2}+\cdots+\beta_{m-1}+\beta_{m} & \leq \alpha_{1}+\alpha_{2}+\cdots+\alpha_{m-1}+\alpha_{m} .
\end{aligned}
\end{gathered}
$$

For any partition $\alpha$ of $\tilde{n}$, and any integer $i \geq 1$, we will denote by $c_{i}(\alpha)$ the numbers of integers $j$ such that $\alpha_{j}=i$. Let $\tilde{n}^{\prime}, \tilde{n}^{\prime \prime}$ two integers such that $\tilde{n}^{\prime}+\tilde{n}^{\prime \prime}=\tilde{n}$, and let $\alpha^{\prime}, \alpha^{\prime \prime}$ be partitions of $\tilde{n}^{\prime}, \tilde{n}^{\prime \prime}$ respectively. We will denote by $\alpha^{\prime} \cup \alpha^{\prime \prime}$ the unique partition of $\tilde{n}$ such that

$$
c_{i}\left(\alpha^{\prime} \cup \alpha^{\prime \prime}\right)=c_{i}\left(\alpha^{\prime}\right)+c_{i}\left(\alpha^{\prime \prime}\right),
$$

for any integer $i \geq 1$, and by $\alpha^{\prime}+\alpha^{\prime \prime}$ the partition of $\tilde{n}$ defined by

$$
\alpha^{\prime}+\alpha^{\prime \prime}:=\left(0 \leq \alpha_{0}^{\prime}+\alpha_{0}^{\prime \prime} \leq \alpha_{1}^{\prime}+\alpha_{1}^{\prime \prime} \leq \ldots \leq \alpha_{m}^{\prime}+\alpha_{m}^{\prime \prime}\right),
$$


Proposition 4.1. Let $\tilde{n}^{\prime}, \tilde{n}^{\prime \prime}$ be such that $\tilde{n}^{\prime}+\tilde{n}^{\prime \prime}=\tilde{n}$. Let $\alpha, \alpha^{\prime}, \alpha^{\prime \prime}$ be partitions of $\tilde{n}, \tilde{n}^{\prime}, \tilde{n}^{\prime \prime}$ respectively. Then the following hold.

(a) Both the representations $E_{\alpha^{\prime} \cup \alpha^{\prime \prime}}$ and $E_{\alpha^{\prime}+\alpha^{\prime \prime}}$ occur with multiplicity one in $I\left(\alpha^{\prime}, \alpha^{\prime \prime}\right)$.

(b) If $E_{\alpha}$ occurs in $I\left(\alpha^{\prime}, \alpha^{\prime \prime}\right)$, then $\alpha^{\prime} \cup \alpha^{\prime \prime} \leq \alpha \leq \alpha^{\prime}+\alpha^{\prime \prime}$.

Proof. For the facts that $E_{\alpha^{\prime} \cup \alpha^{\prime \prime}}$ occurs with multiplicity one in $I\left(\alpha^{\prime}, \alpha^{\prime \prime}\right)$, and that $\alpha^{\prime} \cup \alpha^{\prime \prime} \leq \alpha$, for any $E_{\alpha}$ occurring in $I\left(\alpha^{\prime}, \alpha^{\prime \prime}\right)$, see [26, VIII.2.(5)]. For the remaining assertion, it is enough to notice that $\left(\alpha^{\prime} \cup \alpha^{\prime \prime}\right)^{\vee}=\alpha^{\prime \vee}+\alpha^{\prime \prime \vee}$, that $I\left(\alpha^{\prime}, \alpha^{\prime \prime}\right) \otimes \operatorname{sign}=I\left(\alpha^{\prime \vee}, \alpha^{\prime \prime \vee}\right)$, and that $\alpha \leq \beta$ if and only if $\alpha^{\vee} \geq \beta^{\vee}$, where ${ }^{\vee}$ denotes the dual partition.

\subsection{2.}

First let us introduce the following notation. Let $\alpha$ be a partition of $a$ and let $\beta$ be a partition of $b$, where $a+b=\tilde{n}$. Then we shall set

$$
E_{\alpha, \beta}:=\operatorname{Ind}_{W_{a} \times W_{b}}^{W_{\tilde{n}}}\left(E_{\alpha} \otimes \varphi E_{\beta}\right),
$$

where $W_{a} \times W_{b}$ is regarded as a subgroup of $W_{\tilde{n}}$ in a natural way. We denote by $E_{\alpha, \beta}^{\prime}$ the restriction of $E_{\alpha, \beta}$ to $W_{\tilde{n}}^{\prime}$ when that restriction is irreducible (that is when the sets $\left\{\alpha_{i}\right\}$ and $\left\{\beta_{j}\right\}$ do not coincide); if the restriction is not irreducible we shall write $\operatorname{Res}_{W_{\tilde{n}}^{\prime}}^{W_{\tilde{n}}^{\prime}}\left(E_{\alpha, \beta}\right)=E_{\alpha, \beta}^{\prime}+E_{\alpha, \beta}^{\prime \prime}$.

Proposition 4.2. Let $\tilde{n}^{\prime}, \tilde{n}^{\prime \prime}$ be two integers such that $\tilde{n}^{\prime}+\tilde{n}^{\prime \prime}=\tilde{n}$. Let $\left(a^{\prime}, b^{\prime}\right),\left(a^{\prime \prime \prime}, b^{\prime \prime}\right)$ be two pairs of integers such that $a^{\prime}+b^{\prime}=\tilde{n}^{\prime}, a^{\prime \prime}+b^{\prime \prime}=\tilde{n}^{\prime \prime}$. Let $\alpha^{\prime}, \alpha^{\prime \prime}, \beta^{\prime}, \beta^{\prime \prime}$ be partitions of $a^{\prime}, a^{\prime \prime}, b^{\prime}, b^{\prime \prime}$ respectively. Then

$$
\begin{aligned}
\operatorname{Ind}_{W_{\tilde{n}^{\prime}} \times W_{\tilde{n}^{\prime \prime}}}^{W_{\tilde{n}}}\left(E_{\alpha^{\prime}, \beta^{\prime}} \otimes E_{\alpha^{\prime \prime}, \beta^{\prime \prime}}\right) & \\
\quad= & \sum_{\substack{(\alpha, \beta) \\
\alpha \dashv a^{\prime}+a^{\prime \prime}, \beta \dashv b^{\prime}+b^{\prime \prime}}} m\left(\alpha^{\prime \prime}, \alpha \backslash \alpha^{\prime}\right) m\left(\beta^{\prime \prime}, \beta \backslash \beta^{\prime}\right) E_{\alpha, \beta} .
\end{aligned}
$$

Proof. It follows from $[27, \S 7]$ that $\left.\operatorname{Ind}_{W_{\tilde{n}}^{\prime} \times W_{\tilde{n}^{\prime \prime}}}^{W_{\tilde{n}}}\left(E_{\alpha^{\prime}, \beta^{\prime}} \otimes E_{\alpha^{\prime \prime} \otimes \beta^{\prime \prime}}\right)\right)$ equals

$$
\begin{aligned}
& \operatorname{Ind}_{W_{a^{\prime}+a^{\prime \prime}, b^{\prime}+b^{\prime \prime}}}^{W_{\tilde{n}}}\left(\operatorname{Ind}_{\mathfrak{S}_{a^{\prime}} \times \mathfrak{S}_{a^{\prime \prime}}}^{\mathfrak{S a} a^{\prime}+a^{\prime \prime}}\left(E_{\alpha^{\prime}} \otimes E_{\alpha^{\prime \prime}}\right) \otimes \varphi \operatorname{Ind}_{\mathfrak{S}_{b^{\prime}} \times \mathfrak{S}_{b^{\prime \prime}}}^{\mathfrak{S} b^{\prime}+b^{\prime \prime}}\left(E_{\beta^{\prime}} \otimes E_{\beta^{\prime \prime}}\right)\right) \\
& =\sum_{\substack{\alpha \dashv a^{\prime}+a^{\prime \prime} \\
\beta \dashv b^{\prime}+b^{\prime \prime}}} m\left(\alpha^{\prime \prime}, \alpha \backslash \alpha^{\prime}\right) m\left(\beta^{\prime \prime}, \beta \backslash \beta^{\prime}\right) \operatorname{Ind}_{W_{a+a^{\prime}, b+b^{\prime}}}^{W_{\tilde{n}}}\left(E_{\alpha} \otimes \varphi E_{\beta}\right) \text {. }
\end{aligned}
$$


Corollary 4.3. We keep the notations of Proposition 4.2. Then the following hold.

(a) Both the representations $E_{\alpha^{\prime} \cup \alpha^{\prime \prime}, \beta^{\prime} \cup \beta^{\prime \prime}}$ and $E_{\alpha^{\prime}+\alpha^{\prime \prime}, \beta^{\prime}+\beta^{\prime \prime}}$ occur with multiplicity one in the induced representation

$$
I\left(\alpha^{\prime}, \beta^{\prime} ; \alpha^{\prime \prime}, \beta^{\prime \prime}\right):=\operatorname{Ind}_{W_{\tilde{n}^{\prime}} \times W_{\tilde{n}^{\prime \prime}}}^{W_{\tilde{n}}}\left(E_{\alpha^{\prime}, \beta^{\prime}} \otimes E_{\alpha^{\prime \prime}, \beta^{\prime \prime}}\right) .
$$

(b) Let $\alpha, \beta$ be partitions of $a, b$ respectively. If $E_{\alpha, \beta}$ occurs in $I\left(\alpha^{\prime}, \beta^{\prime} ; \alpha^{\prime \prime}, \beta^{\prime \prime}\right)$, then we have $\alpha^{\prime} \cup \alpha^{\prime \prime} \leq \alpha \leq \alpha^{\prime}+\alpha^{\prime \prime}$ and $\beta^{\prime} \cup \beta^{\prime \prime} \leq$ $\beta \leq \beta^{\prime}+\beta^{\prime \prime}$.

\section{3. $u$-Symbols}

We will now recall some combinatorial objects which have been introduced by Lusztig in [13] in order to parametrize the $\mathcal{N}_{G}$ when $G$ is of classical type.

For an integer $n \geq 1$, let $\Psi_{2 n}=\Psi^{\mathrm{PSp}_{2 n}}$ be the set of all ordered pairs $\left(\begin{array}{l}A \\ B\end{array}\right)$, called $u$-symbols, where $A$ is a finite subset of $\{0,1,2, \ldots\}, B$ a finite subset of $\{1,2,3 \ldots\}$ subject to the condition that

(1) $A, B$ contain no consecutive integers;

$(2)$

$$
\sum_{a \in A} a+\sum_{b \in B} b=n+\frac{(|A|+|B|)(|A|+|B|-1)}{2}
$$

(3) $|A|+|B|$ is odd;

the pairs are taken modulo the equivalence relation generated by the shift operation $\left(\begin{array}{l}A \\ B\end{array}\right) \sim\left(\begin{array}{c}\tilde{A} \\ \tilde{B}\end{array}\right)$ if $\tilde{A}=\{0\} \cup(A+2), \tilde{B}=\{1\} \cup(B+2)$.

Next, for any integer $N \geq 3$, let $\Psi_{N}^{\prime}=\Psi^{\mathrm{SO}_{N}}$ be the set of (unordered) pairs $\left(\begin{array}{l}A^{\prime} \\ B^{\prime}\end{array}\right)$, also called $u$-symbols, where $A^{\prime}$ and $B^{\prime}$ are finite subsets of $\{0,1,2, \ldots\}$ subject to the condition that

(1') $A^{\prime}, B^{\prime}$ contain no consecutive integers, 
$\left(2^{\prime}\right)$

$$
\sum_{a^{\prime} \in A^{\prime}} a^{\prime}+\sum_{b^{\prime} \in B^{\prime}} b^{\prime}=\frac{N}{2}+\frac{\left(\left|A^{\prime}\right|+\left|B^{\prime}\right|-1\right)^{2}-1}{2}
$$

(which implies that $\left.\left|A^{\prime}\right|+\left|B^{\prime}\right| \equiv N \bmod 2\right)$;

the pairs are taken modulo the equivalence relation generated by the shift operation $\left(\begin{array}{l}A^{\prime} \\ B^{\prime}\end{array}\right) \sim\left(\begin{array}{c}\tilde{A}^{\prime} \\ \tilde{B}^{\prime}\end{array}\right)$ if $\tilde{A}^{\prime}=\{0\} \cup\left(A^{\prime}+2\right), \tilde{B}^{\prime}=\{0\} \cup\left(B^{\prime}+2\right)$. Now for each $\theta=\left(\begin{array}{l}A \\ B\end{array}\right) \in \Psi_{N}$ (resp. $\left.\theta=\left(\begin{array}{l}A^{\prime} \\ B^{\prime}\end{array}\right) \in \Psi_{N}^{\prime}\right)$, we define the defect $\operatorname{def}(\theta)$ of $\theta$ by $\operatorname{def}(\theta)=|A|-|B|$ (resp. the absolute value of $\left.\left|A^{\prime}\right|-\left|B^{\prime}\right|\right)$. An element $\left(\begin{array}{l}A^{\prime} \\ B^{\prime}\end{array}\right)$ of $\Psi_{N}^{\prime}$ of zero defect is said to be degenerate if $A^{\prime}=B^{\prime}$. We denote by $\Psi_{N, 0}^{\prime}$ the subset of $u$-symbols of zero defect in $\Psi_{N}^{\prime}$, where the degenerate $u$-symbols are counted twice. We denote by $\Psi_{N, d}\left(\operatorname{resp} . \Psi_{N, d}^{\prime}\right.$, with $d \neq 0)$ the subset of $u$-symbols in $\Psi_{N}\left(\operatorname{resp} . \Psi_{N}^{\prime}\right)$ of defect equal to $d$.

If $G=\mathrm{PSp}_{2 n}$, there exists a bijective map $\psi^{\mathrm{PSp}_{2 n}}: \Psi_{2 n} \longrightarrow \mathcal{N}_{G}$, see $[13,(11.6 .1)]$. If $G=\mathrm{SO}_{N}$, we have a "map" $\psi^{\mathrm{SO}_{N}}: \Psi_{N}^{\prime} \longrightarrow \mathcal{N}_{G}$ which is bijective over the set of non-degenerate $u$-symbols in $\Psi_{N}^{\prime}$ and is such for each degenerate $u$-symbol in $\Psi_{N}^{\prime}$ it has two values, see [13, (11.7.3)].

4.3.1. Orders on $u$-symbols

Let

$$
\theta:=\left(\begin{array}{c}
a_{1}<a_{2}<\cdots<a_{m+d} \\
b_{1}<b_{2}<\cdots<b_{m}
\end{array}\right), \quad \tilde{\theta}:=\left(\begin{array}{c}
\tilde{a}_{1}<\tilde{a}_{2}<\cdots<\tilde{a}_{m+d} \\
\tilde{b}_{1}<\tilde{b}_{2}<\cdots<\tilde{b}_{m}
\end{array}\right)
$$

in $\Psi^{G}$ two $u$-symbols with same defect $d$. We will write $\theta \underset{\Psi^{G}}{\leq} \tilde{\theta}$ if

$$
\left\{\begin{array}{l}
a_{i}+a_{i+1}+\cdots+a_{m+d} \leq \tilde{a}_{i}+\tilde{a}_{i+1}+\cdots+\tilde{a}_{m}, \text { for any } i \in\{0,1, \ldots, m+d\} \\
b_{j}+b_{j+1}+\cdots+b_{m} \leq \tilde{b}_{j}+\tilde{b}_{j+1}+\cdots+\tilde{b}_{m}, \text { for any } j \in\{1,2, \ldots, m\}
\end{array}\right.
$$

\subsubsection{Similarities on $u$-symbols}

Let $\theta_{1}, \theta_{2}$ be two elements of $\Psi_{2 n+1}^{\prime}\left(\operatorname{resp} . \Psi_{2 n}, \Psi_{2 n}^{\prime}\right)$. We say that $\theta_{1}$, $\theta_{2}$ are similar if they can be represented in the form $\left(\begin{array}{l}A_{1} \\ B_{1}\end{array}\right),\left(\begin{array}{l}A_{2} \\ B_{2}\end{array}\right)$ so that $A_{1} \cup B_{1}=A_{2} \cup B_{2}, A_{1} \cap B_{1}=A_{2} \cap B_{2}$. We then write $\theta_{1} \sim \theta_{2}$. This is an equivalence relation on $\Psi_{2 n+1}^{\prime}$ (resp. $\Psi_{2 n}, \Psi_{2 n}^{\prime}$ ) called similarity. In each 
similarity class of $\Psi_{2 n+1}^{\prime}\left(\right.$ resp. $\left.\Psi_{2 n}, \Psi_{2 n}^{\prime}\right)$ there is unique element which can be represented by $\left(\begin{array}{l}A \\ B\end{array}\right)$ with

$$
A=\left\{a_{1}<a_{2}<\cdots<a_{h}\right\}, \quad B=\left\{b_{1}<b_{2}<\cdots<b_{l}\right\}
$$

such that the following holds:

$$
\begin{aligned}
& h=l+1, a_{1} \leq b_{1} \leq a_{2} \leq b_{2} \leq \cdots \leq a_{h} \leq b_{h} \leq a_{h+1}, \text { if }\left(\begin{array}{l}
A \\
B
\end{array}\right) \in \Psi_{2 n+1}^{\prime} \\
& h=l+1, a_{1} \leq b_{1} \leq a_{2} \leq b_{2} \leq \cdots \leq a_{h} \leq b_{h} \leq a_{h+1}, \text { if }\left(\begin{array}{l}
A \\
B
\end{array}\right) \in \Psi_{2 n} \\
& h=l, a_{1} \leq b_{1} \leq a_{2} \leq b_{2} \leq \cdots \leq a_{h} \leq b_{h}, \text { if }\left(\begin{array}{l}
A \\
B
\end{array}\right) \in \Psi_{2 n}^{\prime} .
\end{aligned}
$$

Such an element is said to be distinguished.

4.3.3. Main result on $u$-symbols

Following $[15,(4.4)]$, we define a function

$$
b: \Psi_{2 n+1}^{\prime} \rightarrow \mathbb{N} \quad\left(\operatorname{resp} . b: \Psi_{2 n} \rightarrow \mathbb{N}, \quad b: \Psi_{2 n}^{\prime} \rightarrow \mathbb{N}\right),
$$

as follows.

$\left(B_{n}\right)$ Let $\theta=\left(\begin{array}{l}A \\ B\end{array}\right) \in \Psi_{2 n+1}^{\prime}$. Write the entries of $A$ and $B$ in a single row in increasing order: $x_{0} \leq x_{1} \leq x_{2} \leq \cdots \leq x_{2 m}$. Let $x_{0}^{0} \leq x_{1}^{0} \leq$ $x_{2}^{0} \leq \cdots \leq x_{2 m}^{0}$ be the sequence $0 \leq 0 \leq 2 \leq 2 \leq \cdots \leq 2(m-1) \leq$ $2(m-1) \leq 2 m$. Then by definition

$$
b(\theta):=\sum_{0 \leq i<j \leq 2 m} \inf \left(x_{i}, x_{j}\right)-\sum_{0 \leq i<j \leq 2 m} \inf \left(x_{i}^{0}, x_{j}^{0}\right) .
$$

$\left(C_{n}\right)$ Let $\theta=\left(\begin{array}{l}A \\ B\end{array}\right) \in \Psi_{2 n}$. Write the entries of $A$ and $B$ in a single row in increasing order: $x_{0} \leq x_{1} \leq x_{2} \leq \cdots \leq x_{2 m}$. Let $x_{0}^{0} \leq x_{1}^{0} \leq x_{2}^{0} \leq$ $\cdots \leq x_{2 m}^{0}$ be the sequence $0 \leq 1 \leq 2 \leq 3 \leq \cdots \leq 2 m-1 \leq 2 m$. Then by definition

$$
b(\theta):=\sum_{0 \leq i<j \leq 2 m} \inf \left(x_{i}, x_{j}\right)-\sum_{0 \leq i<j \leq 2 m} \inf \left(x_{i}^{0}, x_{j}^{0}\right) .
$$


$\left(D_{n}\right)$ Let $\theta=\left(\begin{array}{l}A \\ B\end{array}\right) \in \Psi_{2 n}^{\prime}$. Write the entries of $A$ and $B$ in a single row in increasing order: $x_{1} \leq x_{2} \leq \cdots \leq x_{2 m}$. Let $x_{1}^{0} \leq x_{2}^{0} \leq \cdots \leq x_{2 m}^{0}$ be the sequence $0 \leq 0 \leq 2 \leq 2 \leq \cdots \leq 2(m-1) \leq 2(m-1)$. Then by definition

$$
b(\theta):=\sum_{1 \leq i<j \leq 2 m} \inf \left(x_{i}, x_{j}\right)-\sum_{1 \leq i<j \leq 2 m} \inf \left(x_{i}^{0}, x_{j}^{0}\right) .
$$

First note that $b(\theta)$ is indeed well-defined. Furthermore, $b$ is constant on similarity classes, and we have

$$
b(\theta)=d(C(\theta))=\frac{1}{2}(\operatorname{dim} G-\operatorname{dim} C(\theta)-\mathrm{rk} G)
$$

where $(C(\theta), \mathcal{E}(\theta))$ denotes the element of $\mathcal{N}_{G}$ corresponding to $\theta$ via the $\operatorname{map} \psi^{G}$.

It follows from (4.13), that $b(\theta) \geq b(\tilde{\theta})$ if and only if $\operatorname{dim} C(\theta) \leq$ $\operatorname{dim} C(\tilde{\theta})$. Let $\varepsilon$ be equal to 0 in case $B_{n}$ and $C_{n}$, and equal to 1 in case $D_{n}$. Hence we have $\operatorname{dim} C(\theta) \leq \operatorname{dim} C(\tilde{\theta})$ if and only if

$$
\sum_{\varepsilon \leq i<j \leq 2 m} \inf \left(x_{i}, x_{j}\right) \geq \sum_{\varepsilon \leq i<j \leq 2 m} \inf \left(\tilde{x}_{i}, \tilde{x}_{j}\right)
$$

where $x_{\varepsilon} \leq x_{\varepsilon+1} \leq \cdots \leq x_{2 m}$ and $\tilde{x}_{\varepsilon} \leq \tilde{x}_{\varepsilon+1} \leq \tilde{x}_{2} \leq \cdots \leq \tilde{x}_{2 m}$ are the entries of $\theta$ and $\tilde{\theta}$ respectively.

THEOREM 4.4. Let $\theta, \tilde{\theta}$ in $\Psi^{G}$ with same defect. If $\theta \underset{\Psi^{G}}{\leq} \tilde{\theta}$, then $\operatorname{dim} C(\theta) \leq \operatorname{dim} C(\tilde{\theta})$.

Proof. We can assume that $\theta$ and $\tilde{\theta}$ belong to $\Psi_{T}^{H}$. Then we set

$$
\theta:=\left(\begin{array}{c}
A \\
B
\end{array}\right)=\left(\begin{array}{c}
a_{\varepsilon}<a_{\varepsilon+1}<\cdots<a_{m} \\
b_{1}<b_{2}<\cdots<b_{m}
\end{array}\right)
$$

We get

$$
\sum_{\varepsilon \leq i<j \leq 2 m} \inf \left(x_{i}, x_{j}\right)=\sum_{i=\varepsilon}^{m}(m-i) a_{i}+\sum_{j=1}^{m}(m-j) b_{j}+\sum_{\substack{a \in A \\ b \in B}} \inf (a, b),
$$


that is,

$2 m n-\sum_{i=1}^{m}\left(a_{i}+a_{i+1}+\cdots+a_{m}\right)-\sum_{j=1}^{m}\left(b_{j}+b_{j+1}+\cdots+b_{m}\right)+\sum_{\substack{a \in A \\ b \in B}} \inf (a, b)$.

We set $\alpha:=\left(a_{1}<a_{2}<\cdots<a_{m}\right)$ and $\beta:=\left(b_{1}<b_{2}<\cdots<b_{m}\right)$, and similarly $\tilde{\alpha}:=\left(\tilde{a}_{1}<\tilde{a}_{2}<\cdots<\tilde{a}_{m}\right)$ and $\tilde{\beta}:=\left(\tilde{b}_{1}<\tilde{b}_{2}<\cdots<\tilde{b}_{m}\right)$, for

$$
\tilde{\theta}=\left(\begin{array}{c}
\tilde{a}_{\varepsilon}<\tilde{a}_{\varepsilon+1}<\cdots<\tilde{a}_{m} \\
\tilde{b}_{1}<\tilde{b}_{2}<\cdots<\tilde{b}_{m}
\end{array}\right)
$$

If $\theta \underset{\Psi^{G}}{\leq} \tilde{\theta}$, then by definition $\alpha \leq \tilde{\alpha}$ and $\beta \leq \tilde{\beta}$. Hence we clearly have

$$
-\sum_{i=1}^{m}\left(a_{i}+a_{i+1}+\cdots+a_{m}\right) \geq-\sum_{i=1}^{m}\left(\tilde{a}_{i}+\tilde{a}_{i+1}+\cdots+\tilde{a}_{m}\right)
$$

and similarly for the $b, \tilde{b}$.

Moreover, setting

$$
G(\alpha, \beta):=\sum_{\substack{a \in A \\ b \in B}} \inf (a, b)
$$

we check that $G(\alpha, \beta) \geq G(\tilde{\alpha}, \tilde{\beta})$. For this, it is enough to show that

$\left(^{*}\right)$ If $\alpha \leq \tilde{\alpha}$, then we have $G(\alpha, \beta) \geq G(\tilde{\alpha}, \beta)$, and similarly if $\beta \leq \tilde{\beta}$, then $G(\alpha, \beta) \geq G(\alpha, \tilde{\beta})$.

The verification of $(*)$ is reduced to the special case where $\tilde{\alpha}$ is

$$
\left(a_{\varepsilon}<a_{\varepsilon+1}<\cdots<a_{k-1}<a_{k}-1<a_{k+1}<\cdots<a_{l-1}<a_{l}+1<a_{l+1}<\cdots<a_{m}\right) .
$$

But in this case $\left(^{*}\right)$ is easily verified using the definition of $G(\alpha, \beta)$.

Thus, if $\alpha \leq \tilde{\alpha}$ and $\beta \leq \tilde{\beta}$, we have obtained that $b(\theta) \geq b(\tilde{\theta})$, that is, $\operatorname{dim} C(\theta) \leq \operatorname{dim} C(\tilde{\theta})$. 


\subsection{End of the proof of Theorem 1.1}

For $L$ and $\tilde{n}$ as in (4.1.3), and $H$ as in 4.1.7, we take for $\Phi_{T}^{H}$ the set $\Phi_{\tilde{n}, 1}$ of symbols of rank $\tilde{n}$ and defect 1 (see [8]). Let $\Sigma_{L}^{G}: \operatorname{Irr}\left(W_{\tilde{n}}\right) \rightarrow \Phi_{T}^{H}$ be the natural bijection defined in [8, Lemma 2.7]. We associate with any symbol

$$
\Lambda_{T}:=\left(\begin{array}{c}
\lambda_{0}<\lambda_{1}<\cdots<\lambda_{m} \\
\mu_{1}<\mu_{2}<\cdots<\mu_{m}
\end{array}\right) \in \Phi_{T}^{H}
$$

two partitions $\alpha^{\Lambda_{T}}, \beta^{\Lambda_{T}}$ of the following form

$$
\alpha^{\Lambda_{T}}=\left(0 \leq \alpha_{0} \leq \alpha_{1} \leq \cdots \leq \alpha_{m}\right), \quad \beta^{\Lambda_{T}}=\left(0 \leq \beta_{1} \leq \beta_{2} \leq \cdots \leq \beta_{m}\right)
$$

such that $\sum_{i=0}^{m} \alpha_{i}+\sum_{j=1}^{m} \beta_{j}=\tilde{n}$, by setting $\alpha_{i}:=\lambda_{i}-i$ and $\beta_{j}:=\mu_{j}-j+1$. We will write $\Lambda_{T} \widetilde{\Lambda}_{T}$, for $\Lambda_{T}, \widetilde{\Lambda}_{T} \in \Phi_{T}^{H}$, if both $\alpha^{\Lambda_{T}} \leq \alpha^{\widetilde{\Lambda}_{T}}$, and $\beta^{\Lambda_{T}} \leq \beta^{\widetilde{\Lambda}_{T}}$; hence,

$$
\Lambda_{T}=\left(\begin{array}{c}
\lambda_{0}<\lambda_{1}<\cdots<\lambda_{m} \\
\mu_{1}<\mu_{2}<\cdots<\mu_{m}
\end{array}\right) \underset{\Phi_{T}^{H}}{\leq}\left(\begin{array}{c}
\tilde{\lambda}_{0}<\tilde{\lambda}_{1}<\cdots<\tilde{\lambda}_{m} \\
\tilde{\mu}_{1}<\tilde{\mu}_{2}<\cdots<\tilde{\mu}_{m}
\end{array}\right)=\widetilde{\Lambda}_{T}
$$

if and only if

$$
\left\{\begin{array}{l}
\lambda_{i}+\lambda_{i+1}+\cdots+\lambda_{m} \leq \tilde{\lambda}_{i}+\tilde{\lambda}_{i+1}+\cdots+\tilde{\lambda}_{m}, \text { for any } i \in\{0,1, \ldots, m\}, \\
\mu_{j}+\mu_{j+1}+\cdots+\mu_{m} \leq \tilde{\mu}_{j}+\tilde{\mu}_{j+1}+\cdots+\tilde{\mu}_{m}, \text { for any } j \in\{1,2, \ldots, m\} .
\end{array}\right.
$$

By using Corollary 4.3, we see that there exists $E_{\max } \in \operatorname{Irr}\left(W_{L}^{G}\right)$ occurring with mutiplicity one in $\operatorname{Ind}_{W_{L, s}^{G}}^{W_{G}^{G}}\left(E^{\prime}\right)$ such that

$$
\Sigma_{L}^{G}(E) \underset{\Phi_{T}^{H}}{\leq \Sigma_{L}^{G}}\left(E_{\max }\right), \text { for every } E \text { occurring in } \operatorname{Ind}_{W_{L, s}^{G}}^{W_{L}^{G}}\left(E^{\prime}\right) .
$$

Now we set

- $\Theta_{L}^{G}:=\Theta_{\tilde{n}}$ if $d \geq 1\left(\right.$ resp. $\Theta_{L}^{G}:={ }^{t} \Theta_{\tilde{n}}$ if $\left.d \leq-1\right)$, when $G=\operatorname{PSp}_{2 n}$,

- $\Theta_{L}^{G}:=\Theta_{\tilde{n}}^{\prime}$, when $G=\mathrm{SO}_{2 n+1}$,

- $\Theta_{L}^{G}:=\Theta_{\tilde{n}}^{\prime}$, when $G=\mathrm{SO}_{2 n}$ and $t \geq 1$,

where

$\Theta_{\tilde{n}}: \operatorname{Irr}\left(W_{\tilde{n}}\right) \stackrel{1-1}{\longrightarrow} \Psi_{2 \tilde{n}, 1},{ }^{t} \Theta_{\tilde{n}}: \operatorname{Irr}\left(W_{\tilde{n}}\right) \stackrel{1-1}{\longrightarrow} \Psi_{2 \tilde{n}, 1}, \quad \Theta_{\tilde{n}}^{\prime}: \operatorname{Irr}\left(W_{\tilde{n}}\right) \stackrel{1-1}{\longrightarrow} \Psi_{2 \tilde{n}+1,1}^{\prime}$ 
are the bijections defined in $[23, \S 5.7]$. It follows from the explicit descriptions of $\Theta_{L}^{G}$ and $\Sigma_{L}^{G}$ that, for any $\Lambda_{T}, \tilde{\Lambda}_{T}$ in $\Phi_{T}^{H}$,

$$
\Lambda_{T} \underset{\Phi_{T}^{H}}{\leq} \tilde{\Lambda}_{T} \quad \text { if and only if } \quad \sigma_{L}^{G}\left(\Lambda_{T}\right) \underset{\Psi_{T}^{H}}{\leq} \sigma_{L}^{G}\left(\tilde{\Lambda}_{T}\right)
$$

where $\sigma_{L}^{G}$ is the map defined in 4.7 .

Let $\Delta_{L}^{G}: \Psi_{T}^{H} \rightarrow \Psi_{L}^{G}$ be the map defined by Lusztig in [13, $\S 12.2$ and $\S 13.2]$. We observe also that

$$
\theta \underset{\Psi_{T}^{H}}{\leq} \tilde{\theta} \quad \text { if and only if } \quad \Delta_{L}^{G}(\theta) \underset{\Psi^{G}}{\leq} \Delta_{L}^{G}(\tilde{\theta})
$$

for any $\theta$ and $\tilde{\theta}$ in $\Psi_{T}^{H}$. Then the assertion (a) of Theorem 1.1 follows from (4.14) and Theorem 4.4, as explained in Section 4.1.2.

\section{REFERENCES}

[1] D. Alvis and G. Lusztig, On Springer's correspondence for simple groups of type $E_{n}$ $(n=6,7,8)$. With an appendix by N. Spaltenstein, Math. Proc. Cambridge Philos. Soc., 92, no. 1, 65-78.

[2] A.-M. Aubert, Some properties of character sheaves, Pacific J. Math. (Special Issue in the honor of Olga Taussky-Todd) (1998), 37-51.

[3] R.W. Carter, Finite groups of Lie type: Conjugacy classes and complex characters, Wiley, New York, 1985.

[4] P. Deligne and G. Lusztig, Representations of reductive groups over finite fields, Ann. of Math., 103 (1976), 103-161.

[5] M. Geck, On the average value of the irreducible characters of finite groups of Lie type on geometric unipotent classes, Doc. Math., 1, no. 15 (1996), 293-317.

[6] R. Hotta and T.A. Springer, A specialization theorem for certain Weyl group representations and an application to the Green polynomials of unitary groups, Invent. Math., 41, no. 2 (1977), 113-127.

[7] G. Laumon, Faisceaux caractéres (d'aprés Lusztig), Séminaire Bourbaki, Vol. 1988/89. Astérisque No. 177-178, (1989), Exp. No. 709, 231-260.

[8] G. Lusztig, Irreducible representations of finite classical groups, Invent. Math., 43 (1977), 125-175.

[9] _ A class of irreducible representations of a Weyl group, Proc. Kon. Nederl. Akad. series A, 82 (1979), 323-335.

[10] — Green polynomials and singularities of unipotent classes, Adv. Math., 42 (1981), 169-178.

[11] _ A class of irreducible representations of a Weyl group II, Proc. Kon. Nederl. Akad., series A, 85 (1982), 323-335. 
[12] Characters of reductive groups over a finite field Annals Math. Studies vol. 107, Princeton University Press, 1984.

[13] — Intersection cohomology complexes on a reductive group, Invent. Math., $\mathbf{7 5}$ (1984), 205-272.

[14] Character sheaves, Adv. Math. 56 (1985), 193-237, 57 (1985), 226-265, 57 (1985), 266-315, 59 (1986), 1-63, 61 (1986), 103-155.

[15] - On the character values of finite Chevalley groups at unipotent elements, J. Algebra, 104 (1986), 146-194.

[16] _ Green functions and character sheaves, Ann. Math., 131 (1990), 355-408.

[17] — A unipotent support for irreducible representations, Adv. Math., 94 (1992), 139-179.

[18] _ Remarks on computing irreducible characters, J. Amer. Math. Soc., 5 (1992), 971-986.

[19] T. Shoji, On the Springer representations of the Weyl groups of classical algebraic groups, Comm. Algebra, 7 (16) (1979), 1713-1745, 7 (18) (1979), 2027-2033.

[20] $\longrightarrow$ On the Springer representations of Chevalley groups of type $F_{4}$, Comm. Algebra, 8 (1980), 409-440.

[21] - Green functions of reductive groups over a field, Proc. Conf., Arcata/Calif. 1986, Part 2, Proc. of Symposia in Pure Math., 47 (1987), 297-303.

[22] Character sheaves and almost characters of reductive groups I and II, Adv. Math., 111, No. 2 (1995), 244-313, 314-354.

[23] Unipotent characters of finite classical groups. Finite reductive groups (Luminy, 1994), 373-413, Progr. Math., 141, Birkhäuser Boston, Boston, MA, 1997.

[24] N. Spaltenstein, Classes unipotentes et sous-groupes de Borel, Lecture Notes in Math., 946, Springer, Berlin Heidelberg New-York, 1982.

[25] T.A. Springer, Trigonometric sums, Green functions of finite groups and representations of Weyl groups, Invent. Math., 36 (1976), 173-207.

[26] J.-L. Waldspurger, Intégrales orbitales nilpotentes et endoscopie pour les groupes classiques non ramifiés. Astérisque, 269 (2001).

[27] A.V. Zelevinsky, Representations of Finite Classical Groups, Lecture Notes in Math vol. 869, Springer-Verlag, Berlin Heidelberg New-York (1981).

Institut de Mathématiques de Jussieu

U.M.R. 7586 du C.N.R.S.

Projet Formes Automorphes

175 rue du Chevaleret

F-75013, Paris

France

aubert@math.jussieu.fr 